\title{
Image Denoising Research Based on Extreme Filtering
}

\author{
Lihua SUN ${ }^{\mathrm{a}}$, Enliang ZHAO ${ }^{\mathrm{b}}$ \\ Shenyang Jianzhu University, Shenyang, 110168, China \\ aemail:slh0330@163.com, bemail:kuku0319@163.com
}

Keywords: Median Filtering, Impulse Noise, Extreme Filtering, Image Denoising

\begin{abstract}
Median filtering algorithm can make the edge details of the image fuzzy when it is used to remove noise of an image. In this paper a method based on extreme filtering is put forward for image denoising. Firstly extreme filtering algorithm is used to determine whether a pixel is a signal point or a suspicious noise point in a small $3 \times 3$ window, then the same extreme filtering algorithm is used in an expanded $5 \times 5$ window. The suspicious noise points are further defined as signal points and noise points with some pixels in the small window. Median filtering is used to process the noise points while the original pixel values are retained for the signal points. This method is used in numerical experiments. The results show that the proposed method for image denoising can effectively avoid fuzzy image while texture details of image is due to the loss and can obtain relatively clearer image. The denoised image can retain the image edges of the image and can improve the peak signal-to-noise ratio.
\end{abstract}

\section{Introduction}

During the process of formation, transmission, reception and processing, an image is due to be disturbed by external interference, and impulse noise is generated. It is always one of the basic tasks of image preprocessing that it can effectively remove noise, preserve the integrity and edge information of the original image and can improve the signal-to-noise ratio[1-5]. There are many kinds of noises in the image, and impulse noise is the most common kind of noise. The median filtering method is widely used in the field of image processing, it can reserve the image details while it is used to remove the impulse noise. However, the effect for denoising is affected by the size of the filtering window. The small window can better preserve details of the image, but it cannot effectively remove the noise. Large window can effectively restrain the noise, but it can destroy more details of the image, and result in blurred image. Obviously, we hope the filtering algorithm should only process the noise point, and keep the gray value of the signal point unchanged. Therefore, before the noise is filtered, a detection process of the noise point is needed to separate the noise point from the signal point.

In order to avoid the shortcomings of the median filtering, some scholars have put forward various of improved algorithms, such as adaptive median filtering [6-9], weighted median filtering, switch median filtering [10], median and extremum filtering [11], image denoising based on dynamic window [12]. These algorithms have made useful exploration in improving the performance of the median filtering, but they all have their own limitations in practical applications. A switching median filtering has good effect when noise density is low, and its performance is close to the standard median filtering when the signal-to-noise ratio of the input image gradually decreases. The switch filtering is a cyclic operation, and has a long execution time. At the same time it needs to estimate parameters ahead of time. Hence it is limited in practical applications. In extremum and median filtering, maximum and minimum values are considered as the noise points so that they are filtered out. Thus too many details of the image are lost, and will result in blurred image.

In this paper an improved median filtering algorithm is proposed to remove the impulse noise of the image, and a method based on extreme filtering is proposed for image denoising. Firstly extreme filtering algorithm is used to determine whether a pixel is a signal point or a suspicious noise point in a small $3 \times 3$ window, then the same extreme filtering algorithm is used in an expanded $5 \times 5$ window. The suspicious noise points are further defined as signal points and noise 
points with some pixels in the small window. Median filtering is used to process the noise points while the original pixel values are retained for the signal points. The method can not only effectively distinguish signal points and impulse noise points in the image, and improve signal-to-noise, but also it can effectively protect the edge information of the image, and decrease obscure image by filtering.

\section{The Principle of Median Filtering Algorithm}

Median filtering is proposed by Turky in1971. The principle of median filtering is that a value of a point in the sequence or digital image is taken the place of the median value of the points in the neighborhood.

One dimensional median filtering is defined as follows:

The $n$ number $x_{1}, x_{2}, \cdots, x_{n}$ is arranged according to the size of the value as follows:

$$
x_{i 1} \leq x_{i 2} \leq \cdots \leq x_{\text {in }}
$$

thus

$$
y=\operatorname{Med}\left\{x_{1}, x_{2}, \cdots, x_{n}\right\}= \begin{cases}x_{i((n+1) / 2)} & n \text { is odd }, \\ \frac{1}{2}\left[x_{i(n / 2)}+x_{i(n / 2+1)}\right] & n \text { is even. }\end{cases}
$$

Where $y$ is defined as a median value of the sequence.

Two-dimensional median filtering is defined as follows:

Let $\left\{x_{i, j},(i, j) \in I^{2}\right\}$ be the gray value of the pixel in a digital image and let $A$ be a filtering window whose size is $N=(2 K+1) \times(2 K+1)$, thus

$$
y(i, j)=\operatorname{Med}_{A}\left\{x_{i, j}\right\}=\operatorname{Med}\left\{x_{(i+r),(j+s)},(r, s) \in A(i, j) \in I^{2}\right\} .
$$

For the two-dimensional median filtering, some form of two-dimensional window may be adopted such as: linear, circle, square, cross, circular and etc. Generally the $3 \times 3$ square window is used which can be gradually increased to a $5 \times 5$ square window, $7 \times 7$ square window, until the filtering effect is satisfied.

Traditional median filtering algorithm processes all the pixels in the image by unfied median method. The method loses some of the original information of the image while it removes the noise. It is especially serious that the edge details of image are more fuzzy. The larger the filtering window is the more obvious the influence is. If we can accurately judge the signal points and noise points in the image,we can only handle noise points, and keep the signal points unchanged. Thus it can reduce the fuzzy edge details in the image, does not affect signal points in the filtering process, and only play the role of removing impulse noise.

\section{Improved Median Filtering}

The median filtering algorithm often needs to handle all pixel points by unified method for image denosing. At the same time the noise points are removed, some non-noise points with noise points (e.g. edges) are processed in the image, which makes fuzzy image. For this reason the proposed method begins with judgment for noise points in this paper. Then noise points are handled by improved median filtering algorithm, the non-noise points are retained. The gray values between adjacent pixels in the image have great relevance. That is to say, if the pixels and their neighboring pixels are judged for impulse noise points. It is a very small possibility that all the pixels are in fact impulse noise points. It is possible that these values of pixels are very close to the edges of image, or a smooth region. Thus first of all we can make use of extreme filtering to judge the pixel between a signal point and a suspicious noise point in the image. For the suspicious noise point we judge the signal point or the impulse noise point in the enlarged small window by extreme filtering algorithm. On the basis of the above method, the image will be dealed with as follows:

Step1: In the noisy image we move $3 \times 3$ filtering window in order that the center of filtering window is associated with some a pixel point. Then we may determinate the gray value of the pixel 
for the maximum or the minimum value. If it is the maximum or minimum value, the point is considered as a suspicious noise point to enter the next step of detection. On the contrary, it is considered as the signal point to keep unchanged.

Step2: The filtering window is expanded to $5 \times 5$, we may further determinate the gray value of the pixel (except 8 pixels in $3 \times 3$ filtering window) which is a maximum or a minimum value. If it is not the maximum or minimum value, it is considered that the point is the signal point, and its gray value will be output.

Step3: If the gray value of the pixel is judged to be a maximum or a minimum, the point is considered as a noise point. The median value of 8 pixel values in the $3 \times 3$ filtering window is output instead of the original gray value.

This method avoids that all pixels are handled by unified median method, but also avoids that the maximum and minimum values are considered as noise points that are filtered out by the extremum and median filtering. As a result it can effectively keep the edge feature of the image and avoid the fuzzy image edges.

\section{Experimental Results and Discussion}

Matlab7.0 software is used to implement the algorithm. In the experiment a $256 \times 256$ Lena map is adopted as an example. Figure 1 shows images of Lena by 3 algorithms for the removal of impulse noise. Figure 2 shows the PSNR of the output image by 3 different algorithms. Figure 3 shows the mean square error of different filtering methods. Table 1 shows the PSNR for image denosing by 3 filtering algorithms with impulse noise of different intensity. Table 2 shows the relation between mean square error of the output image and noise density of the original input image by 3 algorithms.

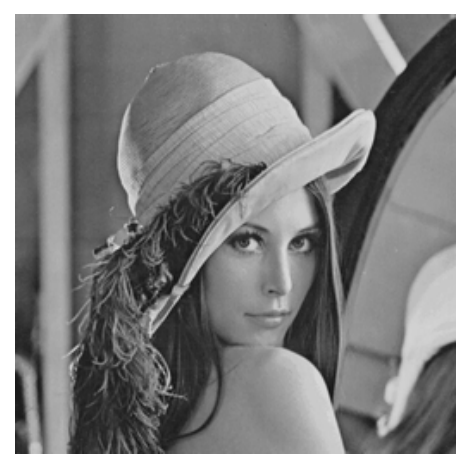

(a)The original image

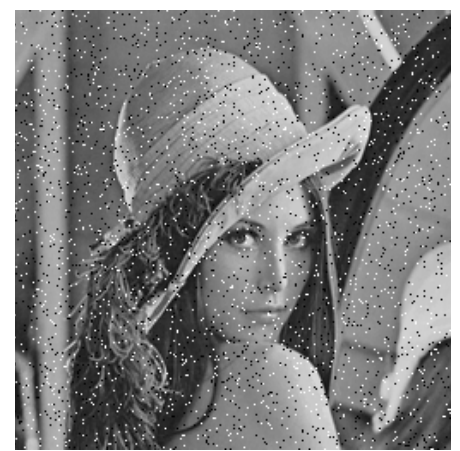

(b)The image with 5\%

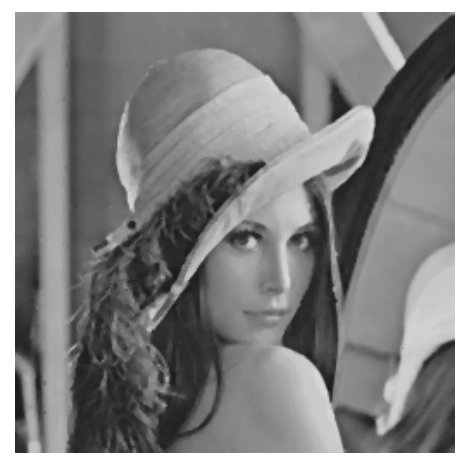

(c)The image by median filtering in a $3 \times 3$ window

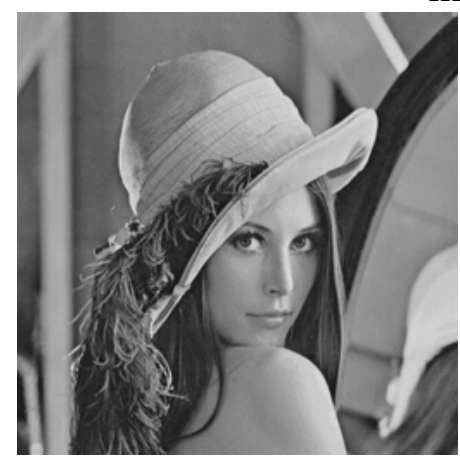

(d) The denoised image by extremum filtering impulse noise

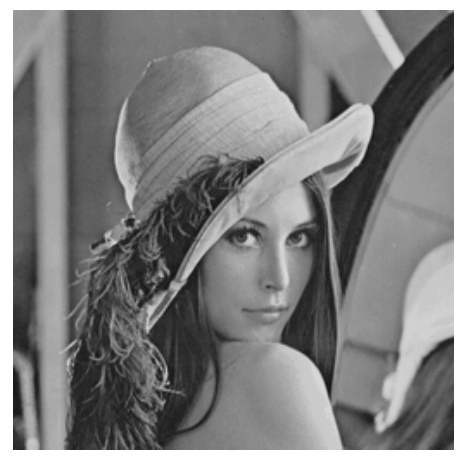

(f) The denoised image by the proposed method

Fig.1 The image by three different methods for the removal of impulse noise 


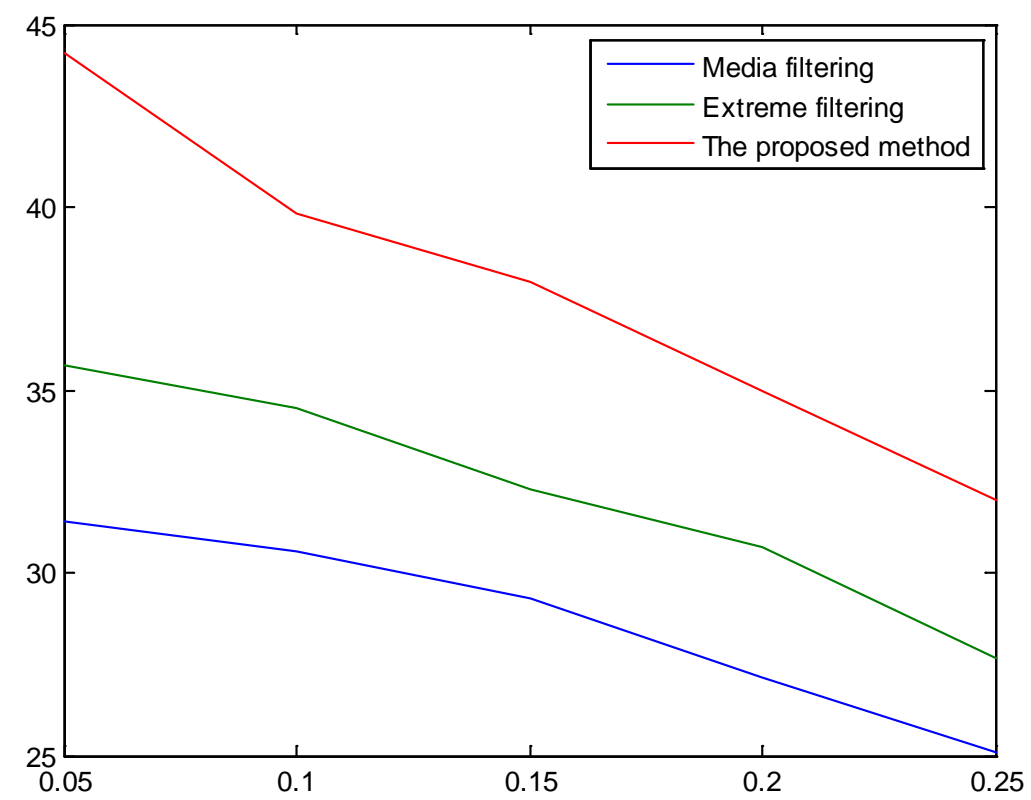

Fig.2 The PSNR by three different filtering methods

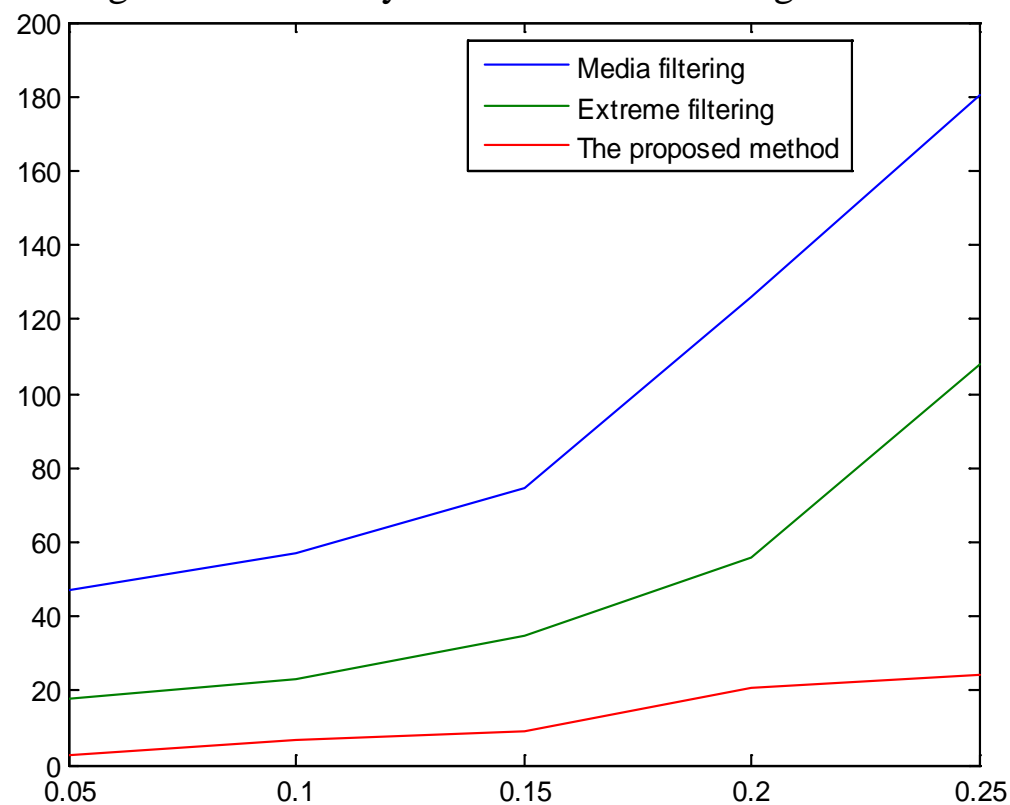

Fig.3 The mean square error by three different filtering methods

Table1 The PSNR by 3 filtering algorithms with impulse noise of different intensity

\begin{tabular}{|c|c|c|c|c|c|c|}
\hline \multirow[b]{2}{*}{ method } & \multicolumn{2}{|c|}{ Test I } & \multicolumn{2}{|l|}{ Test II } & \multicolumn{2}{|l|}{ Test III } \\
\hline & tensity & PSNR & noise intensity & PSNR & noise intensity & PSNR \\
\hline median filtering & 0.05 & 31.42 & 0.1 & 30.57 & 0.2 & 27.12 \\
\hline extremum filtering & 0.05 & 35.65 & 0.1 & 34.51 & 0.2 & 30.68 \\
\hline the proposed method & 0.05 & 44.22 & 0.1 & 39.81 & 0.2 & 34.99 \\
\hline
\end{tabular}

Table2 The relation between mean square error and noise density by 3 filtering algorithms

\begin{tabular}{|c|c|c|c|c|c|c|}
\hline \multirow[b]{2}{*}{ method } & \multicolumn{2}{|c|}{ Test I } & \multicolumn{2}{|c|}{ Test II } & \multicolumn{2}{|c|}{ Test III } \\
\hline & noise inte & y MSER & noise i & y MSEF & noise il & MSER \\
\hline median filtering & 0.05 & 46.90 & 0.1 & 56.97 & 0.2 & 126.12 \\
\hline extremum filter & ing 0.05 & 17.72 & 0.1 & 23.00 & 0.2 & 55.58 \\
\hline the proposed $\mathrm{m}$ & ethod 0.05 & 2.460 & 0.1 & 6.970 & 0.2 & 20.58 \\
\hline
\end{tabular}

The experiment shows that the proposed algorithm has better effect on the removal of the impulse noise than traditional median filtering algorithm. It can effectively distinguish noise points and signal points, especially can distinguish the edge information of the image and noise points. It 
can accelerate the running speed of the image and improve the PSNR. A clearer image may be got that can preserve details of the image. It is an effective algorithm for image denosing.

\section{Conclusion}

Extreme filtering algorithm is used to determine whether a pixel is a signal point or a suspicious noise point in a small region. The suspicious noise points are further defined as signal points and noise points with neighbour pixels. Median filtering is used to process the noise points while the original pixel values are retained for the signal points. The method can effectively make a distinction between noise points and signal points, especially for the edge information of the image and noise points. A clearer image may be got that can preserve details of the image. The experiment shows that the proposed algorithm has better effect on the removal of the impulse noise than traditional median filtering algorithm.

\section{References}

[1] Jeong B, Lee Y H. Design of weighted order statistic filter using the perception Algorithm [J] . IEEE Transactions on Signal Processing, 1994, 42(11):3264-3269.

[2] Chan R H, Ho C W, Nikolova M. Salt-and-pepper removal by median-type noise detectors and detail-preserving regularization [J]. IEEE Trans. Image processing, 2005,14(10):1479-1485.

[3] Wang Zhou, Zhang David. Progressive switching median filter for the removal of impulse noise from highly corrupted images [J]. IEEE Transactions on Circuits and Systems, 1999, 46 (1):78-80.

[4] Wang Junghua, Lin Lianda. Improved median filter using min/max algorithm for image processing[J]. Electronics Letters, 1977, 33(16):1362-1363.

[5] Sun Lihua, Zhao Enliang, Ma Long. A filtering algorithm for removing impulse noise [J]. Journal of Shenyang Jianzhu University, 2012, 28 (6):1148-1152.

[6] Wang XiaoKai, Li Feng. Improved adaptive median filter [J]. Computer Engineering and Applications, 2010, 46 (3): 175-176.

[7] Wang Min, Cheng Jing, Zhang Fan. An improved adaptive median filtering algorithm [J]. Image Processing, 2010, 26 (4):109- 110.

[8] Xie Jianbin, Liu Tong, Ren Yong. An adaptive fast filtering algorithm for restraining impulse noise [J]. Journal of Image and Graphics, 2009, 14 (5):843- 847.

[9] GaoKefang,Guo Jiangang. An adaptive median filtering method based on noise detection [J]. Journal of Fujian Agriculture and Forestry University: Natural Science, 2009, 38 (3): 333-336.

[10] Xu Chao,Chen Yihu. A switching median filter based on fourfold division for noise detection [J]. Computer Engineering and Design, 2008, 29 (18):4765-4767.

[11] Xing Zangju, Wang Shouju, Deng Haojiang. A new filtering algorithm based on extremum and median value) [J]. Journal of Image and Graphics, 2001, 6 (6):533-536.

[12] Hu Weizhe, Zhao Enliang, Sun Lihua. A median filtering method on impulse noise detection based on dynamic window [C].The 11th Shenyang Science Conference, 2014. 